\title{
Control of Fast Scale Bifurcations in Power-Factor Correction Converters
}

\author{
Damian Giaouris, Member, IEEE, Soumitro Banerjee, Senior Member, IEEE, Bashar Zahawi, Senior Member, IEEE, \\ and Volker Pickert, Member, IEEE
}

\begin{abstract}
This brief proposes a novel controller which greatly enhances the performance of a power-factor correction converter. This controller is optimally tuned to place the eigenvalues of the system well inside the unit circle and hence it guarantees stable operation over a wide range of input voltages. The design of the controller is based on the stability analysis of the system using the state transition matrix over a clock cycle. It is shown that the transition matrix across the switching manifold greatly influences the system's performance, allowing the system to be stabilized by periodically altering the manifold. The results are validated by analytical and numerical studies.
\end{abstract}

Index Terms-Bifurcation, control of power electronics, powerfactor correction (PFC).

\section{INTRODUCTION}

$\mathbf{P}$ OWER-FACTOR correction (PFC) converters are widely used in practical power supplies to regulate the output and to provide an acceptable ratio of active and apparent power. The supply voltage ( $\mathrm{AC}$ at $50 / 60 \mathrm{~Hz}$ ) is first rectified by using a diode bridge rectifier and then the de voltage is supplied to a current mode controlled dc-dc converter (Fig. 1). The demanded current is derived from the rectified voltage and hence, assuming satisfactory control of the dc-dc converter, nearly unity power-factor operation is achieved [1], [2]. The rectified current consists mainly of two frequency components-one at twice the frequency of the line signal and the other a high frequency component related to the clock used by the dc-dc converter. Historically, boost converters working in continuous conduction mode (CCM) or in discontinuous conduction mode (DCM) have been employed using average or peak current control strategies. Isolated PFC converters working in DCM have also been studied [3].

Since the system involves switching, the overall model is piecewise-smooth, and both smooth and nonsmooth bifurcations may occur [4], [5]. In the study of dc-dc converters, the terms fast-scale and slow-scale bifurcation have been coined: the former refers to the instability that affects the dynamics at clock frequency, and the later refers to the generation of a slower frequency of oscillation [6]. In PFC systems, both types of bifurcations have been observed [3], [7]-[9]— which have been

Manuscript received November 1, 2006; revised February 9, 2007. The work of S. Banerjee was supported in part by the Department of Atomic Energy, Government of India, under Project 2003/37/11/BRNS. This paper was recommended by Associate Editor M. di Bernardo.

D. Giaouris, B. Zahawi, and V. Pickert are with the School of Electrical, Electronic and Computer Engineering, University of Newcastle upon Tyne, NE1 7RU, U.K. (e-mail: Damian.Giaouris@ @ncl.ac.uk).

S. Banerjee is with the Centre for Theoretical Studies and Department of Electrical Engineering, Indian Institute of Technology, Kharagpur 721302, India (e-mail: soumitro@iitkgp.ac.in).

Digital Object Identifier 10.1109/TCSII.2007.900350

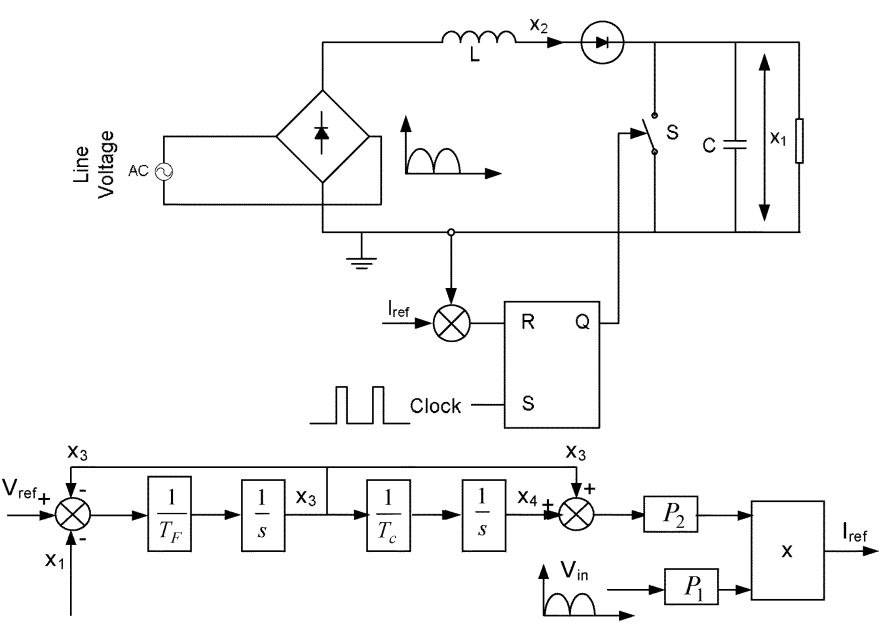

Fig. 1. Boost converter under current peak control.

shown to reduce the power factor of the circuit, thus downgrading the overall performance.

Conventionally these bifurcations are analyzed by using a small signal model of the system [10] or based on the Poincaré map of the periodic orbit. In this brief, the fast scale bifurcations are studied using a different approach based on the state transition matrix of the system over one clock cycle [11], also referred to as the monodromy matrix [12]. Since the system under study involves switches, the influence of the switching manifold has to be taken into account when the monodromy matrix is obtained. This is accomplished by using the state transition matrix across the switching manifold [13], called the saltation matrix. By using this approach it is possible to locate the bifurcation point by calculating the Floquet multipliers.

In this brief, we show that the saltation matrix is mainly responsible for changes in the Floquet multipliers and hence can have a major influence on the stability of the system. This idea has been previously employed in stabilizing discontinuous mechanical systems [14]-[16] where a new and appropriately chosen discontinuity is introduced into the system. In this brief, we propose a novel controller that aims at appropriately altering the saltation matrix so that stable operation is obtained over almost a full line cycle. This is achieved by introducing an additional sinusoidal waveform to the reference current. Earlier it was empirically observed that a sinusoidal perturbation can stabilize the system [17]. In this brief, we analytically explain why the technique works, and develop methods for obtaining optimal choice for the perturbation signal.

A peak current controlled boost converter operating under $\mathrm{CCM}$ - which is known to exhibit fast scale bifurcation [9] - is used to demonstrate the new method of analysis and control. 


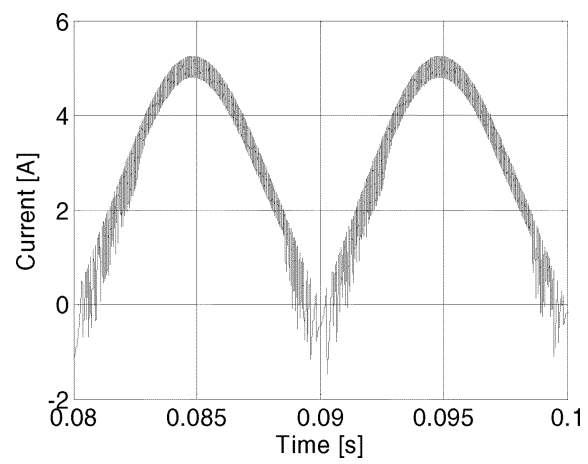

Fig. 2. Response of PFC for $V_{\text {in }}=110 \mathrm{~V}$ RMS $/ 50 \mathrm{~Hz}, V_{\text {ref }}=220 \mathrm{~V}$, $L=2 \mathrm{mH}, C=470 \mu \mathrm{F}, R=135 \Omega$, Clock period $T=20 \mu \mathrm{s}, T_{F}=4 \mathrm{~ms}$, $T_{c}=1 / 70 \mathrm{~s}, P_{2}=1 / 60, P_{1}=0.08$.

\section{PFC BOOST CONVERTER}

The boost converter used in the PFC is shown in Fig. 1. To achieve near unity power factor the demanded current is in phase with the demanded voltage. The demanded voltage $\left(V_{\text {ref }}\right)$ is compared with the capacitor voltage $\left(x_{1}\right)$ and the error signal is filtered using a typical first-order low-pass filter (time constant: $T_{F}$ ) to remove any unwanted noise. The filtered error is fed to a conventional PI controller $\left(K_{P}=P_{2}, K_{I}=P_{2} / T_{c}\right)$ and the output is multiplied by the rectified input voltage times a proportionality constant $P_{1}$ [9]. The total state-space model is

$$
\dot{\mathbf{x}}= \begin{cases}\mathbf{f}_{-}(\mathbf{x}), & \mathrm{S} \text { is on } \\ \mathbf{f}_{+}(\mathbf{x}), & \mathrm{S} \text { is off }\end{cases}
$$

where

$$
\mathbf{f}_{-}(\mathbf{x})=\left[\begin{array}{c}
\frac{-x_{1}}{R C} \\
\frac{V_{i n}}{L} \\
\frac{V_{\text {ref }}-x_{1}-x_{3}}{T_{F}} \\
\frac{x_{3}}{T_{c}}
\end{array}\right], \mathbf{f}_{+}(\mathbf{x})=\left[\begin{array}{c}
\frac{x_{2}}{C}-\frac{x_{1}}{R C} \\
\frac{V_{\text {in }}}{L}-\frac{x_{1}}{L} \\
\frac{V_{\text {ref }}-x_{1}-x_{3}}{T_{F}} \\
\frac{x_{3}}{T_{c}}
\end{array}\right]
$$

where $x_{1}$ is the capacitor voltage and $x_{2}$ is the inductor current.

The two extra states $x_{3}$ and $x_{4}$ are related to the control loop (Fig. 1). The reference current for the peak current controller $\left(I_{\text {ref }}\right)$ is obtained as

$$
I_{\text {ref }}=\left(x_{3}+x_{4}\right) P_{1} P_{2} V_{\text {in }} .
$$

A typical response over a full line cycle is shown in Fig. 2, where it is obvious that for high values of the supply voltage the system is stable while for low values the output current waveform is distorted-which degrades the system performance. Since the line frequency is a few orders of magnitude smaller than the switching frequency, it can be assumed that the supply voltage is constant over one switching cycle [3]. This widely made assumption is of course an approximation which will cause some inaccuracies in the overall analysis. Nevertheless the fundamental properties of the system are retained, as demonstrated in [9]. Hence, the converter is studied assuming constant supply for a range of voltages $V_{\text {in }}$ from 0 to $110 \sqrt{2} \mathrm{~V}$ (the peak value of the rectified voltage). Fig. 3 shows how the system loses stability when the supply voltage is progressively reduced. The values chosen are $V_{\text {in }}=k \times 110 \sqrt{2}, k=1,0.8$,

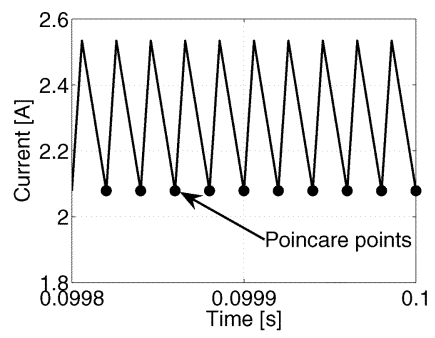

(a)

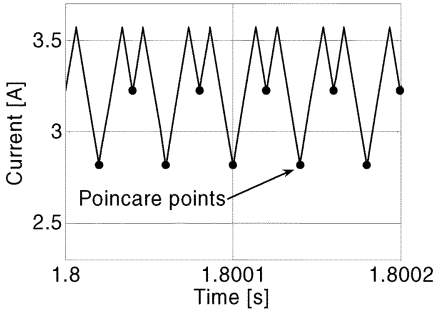

(c)

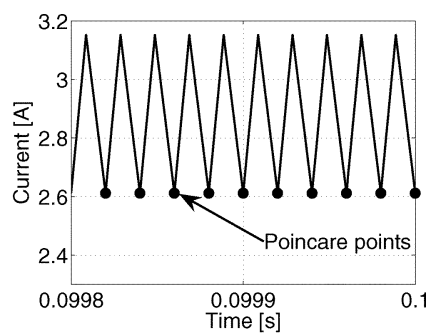

(b)

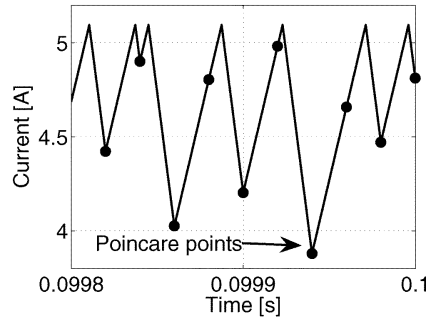

(d)
Fig. 3. The inductor current waveforms for $V_{\text {in }}=k \times 110 \sqrt{2} \mathrm{~V}, k=$ (a) 1.0 , (b) 0.8 , (c) 0.707 , (d) 0.5 . The solid circles are the points of discrete observation taken by sampling the state every $T$ s.

0.7 , and 0.5 . Matlab/Simulink were used for all simulations, using the differential equation solver ode 45 , with maximum step size of $1 \mu \mathrm{s}$ and relative tolerance of $1 \mathrm{~ms}$. For all other parameters, default settings were used.

\section{StabiLity ANALYSiS}

\section{A. Derivation of the Monodromy Matrix}

In this section we obtain the state transition matrix over a complete clock cycle, called the monodromy matrix. The eigenvalues of the monodromy matrix are called Floquet multipliers [12] and are identical to the eigenvalues of the fixed point of the Poincaré map, and hence they determine the stability of the orbit.

For a period-1 orbit in CCM, the state vector follows two smooth vector fields during the on and off periods, and the switching manifold $(h)$ is defined as $h(\mathbf{x}(t))=x_{2}-I_{\text {ref }}$, where $I_{\text {ref }}$ is given by (2). The state transition matrix during the on period is given by $\boldsymbol{\Phi}_{-}(t, 0)=e^{\mathbf{A}_{-} t}$ and during the off period by $\boldsymbol{\Phi}_{+}(t, d T)=e^{\mathbf{A}_{+}(t-d T)}$, where $d$ is the duty cycle of the converter. The state matrices before and after the switching can be easily obtained from the two smooth vector fields

$$
\begin{aligned}
& \mathbf{A}_{-}=\left[\begin{array}{cccc}
-\frac{1}{R C} & 0 & 0 & 0 \\
0 & 0 & 0 & 0 \\
-\frac{1}{T_{F}} & 0 & -\frac{1}{T_{F}} & 0 \\
0 & 0 & \frac{1}{T_{c}} & 0
\end{array}\right] \\
& \mathbf{A}_{+}=\left[\begin{array}{cccc}
-\frac{1}{R_{C}} & \frac{1}{C} & 0 & 0 \\
-\frac{1}{L} & 0 & 0 & 0 \\
-\frac{1}{T_{F}} & 0 & -\frac{1}{T_{F}} & 0 \\
0 & 0 & \frac{1}{T_{C}} & 0
\end{array}\right] .
\end{aligned}
$$

The transition at the switching event is described by the saltation matrix [13] given by

$$
\mathbf{S}=\mathbf{I}_{4}+\frac{\left(\mathbf{f}_{+}-\mathbf{f}_{-}\right) \mathbf{n}^{T}}{\mathbf{n}^{T} \mathbf{f}_{-}+\frac{\partial h}{\partial t}}
$$


where $\mathbf{I}_{4}$ is the identity matrix of order 4 , and $\mathbf{n}$ is the vector normal to the switching manifold given by the gradient of $h$

$$
\mathbf{n}=\nabla h=\left[\begin{array}{llll}
0 & 1 & -P_{1} P_{2} V_{\text {in }} & -P_{1} P_{2} V_{\text {in }}
\end{array}\right]^{T} .
$$

All the terms have to be evaluated at the switching instant $t=$ $d T$.

By using the basic properties of the state transition matrix we can describe the monodromy matrix of the system as

$$
\mathbf{M}=\underline{\boldsymbol{\Phi}}_{+}(T, d T) \mathbf{S} \underline{\boldsymbol{\Phi}}_{-}(d T, 0) .
$$

In the next section we will locate the period-1 limit cycle for all values of the supply voltage and we will use this information to calculate the monodromy matrix and its eigenvalues.

\section{B. Location of the Period-1 Limit Cycle}

In order to locate the limit cycle for different values of $V_{\text {in }}$, we need to know the value of the duty cycle for the stable or unstable period-1 orbit for every value of $V_{\text {in }}$ [11]. The expression for the steady state duty cycle of the boost converter is given by $d=1-V_{\text {in }} / V_{\text {ref }}$ [9]. The state vectors at $t=d T$ and $t=T$ are given by

$$
\begin{aligned}
\mathbf{x}(d T) & =\boldsymbol{\Phi}_{-}(d T, 0) \mathbf{x}(0)+\int_{0}^{d T} \boldsymbol{\Phi}_{-}(d T-\tau, 0) \mathbf{R} d \tau \\
\mathbf{x}(T) & =\boldsymbol{\Phi}_{+}(T, d T) \mathbf{x}(d T)+\int_{d T}^{T} \boldsymbol{\Phi}_{+}(T-\tau, d T) \mathbf{R} d \tau \\
& =\mathbf{x}(0)
\end{aligned}
$$

To simplify the analysis we will use only the first three states of the system as the fourth state does not influence the other three. Hence, the order of the two state matrices will be reduced to 3 , and the input vector $\mathbf{R}$ is given by

$$
\mathbf{R}=\left[\begin{array}{lll}
0 & \frac{V_{\text {in }}}{L} & \frac{V_{\text {ref }}}{T_{F}}
\end{array}\right]^{T} .
$$

By substituting (7) into (8) and by solving for $\mathbf{x}(0)$ we get

$\mathbf{x}(0)=\left(\mathbf{I}_{3}-\boldsymbol{\Phi}_{+}(T, d T) \boldsymbol{\Phi}_{-}(d T, 0)\right)^{-1}\left(\boldsymbol{\Phi}_{+}(T, d T) \mathbf{I}_{-}+\mathbf{I}_{+}\right)$.

where $\mathbf{I}_{-}$and $\mathbf{I}_{+}$are the two integrals in (7) and (8). If the duty cycle is known, (9) gives the state vector at $t=0$, and (7) gives the values of the three states at $t=d T$. For a satisfactory peak current controller, at $t=d T$ we have $x_{2}(d T)=I_{\text {ref }}$. From (2) we can find the value of the fourth state at $t=d T$ as $x_{4}(d T)=-x_{3}(d T)+I_{\text {ref }} /\left(P_{1} P_{2} V_{\text {in }}\right)$.

\section{Evaluation of the Floquet Multipliers}

Thus, we can effectively locate the period-1 (stable or unstable) limit cycle for various values of $V_{\text {in }}$, and can use the monodromy matrix to determine its stability. From (5), we have

$$
\mathbf{f}_{+}-\mathbf{f}_{-}=\left[\begin{array}{c}
\frac{x_{2}}{C} \\
-\frac{x_{1}}{L} \\
0 \\
0
\end{array}\right]
$$

TABLE I

FLOQUET MULTIPLIERS FOR VARIOUS SUPPLY VOLTAGES.

\begin{tabular}{|c|c|}
\hline $\mathrm{k}=1$ & $\mathrm{k}=0.8$ \\
\hline$\left[\begin{array}{c}-0.41413 \\
0.9985 \\
0.9979 \pm 0.0049 j\end{array}\right]$ & {$\left[\begin{array}{c}-0.7679 \\
0.9983 \\
0.9980 \pm 0.0036 j\end{array}\right]$} \\
\hline \hline $\mathrm{k}=0.7$ & $\mathrm{k}=0.5$ \\
\hline$\left[\begin{array}{c}-1.0205 \\
0.9981 \\
0.9981 \pm 0.0028 j\end{array}\right]$ & {$\left[\begin{array}{c}-1.8287 \\
0.9967 \\
0.9988 \pm 0.0014 j\end{array}\right]$} \\
\hline
\end{tabular}

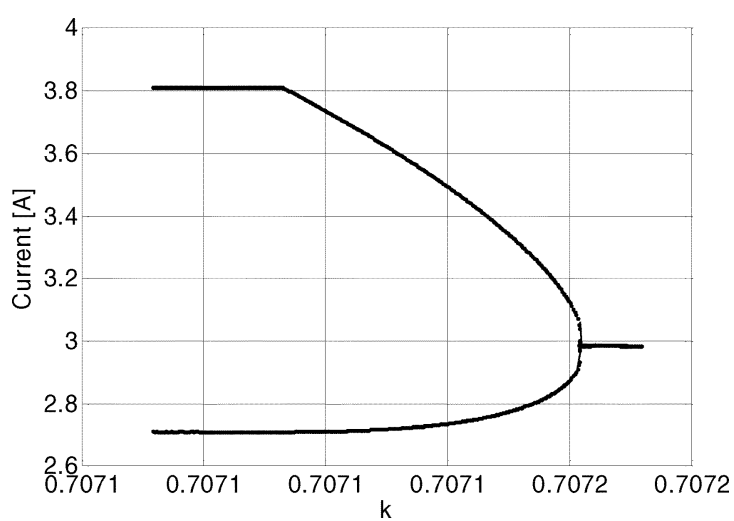

Fig. 4. Bifurcation diagram of the PFC when the input voltage is used as the bifurcation parameter. The value $k=1$ corresponds to the peak voltage of the original rectified signal.

Hence

$$
\begin{aligned}
\left(\mathbf{f}_{+}-\mathbf{f}_{-}\right) \mathbf{n}^{T} & =\left[\begin{array}{cccc}
0 & \frac{x_{2}}{C} & -\frac{x_{2}}{C} P_{1} P_{2} V_{\text {in }} & -\frac{x_{2}}{C} P_{1} P_{2} V_{\text {in }} \\
0 & -\frac{x_{1}}{L} & \frac{x_{1} P_{1} P_{2} V_{\text {in }}}{L} & \frac{x_{1} P_{1} P_{2} V_{\text {in }}}{L} \\
0 & 0 & 0 & 0 \\
0 & 0 & 0 & 0
\end{array}\right] \\
\mathbf{n}^{T} \mathbf{f}_{-} & =\frac{V_{\text {in }}}{L}-P_{1} P_{2} V_{\text {in }} \frac{V_{\text {ref }}-x_{1}-x_{3}}{T_{F}}-P_{1} P_{2} V_{\text {in }} \frac{x_{3}}{T_{c}}
\end{aligned} .
$$

Since the switching manifold does not vary with time, $\partial h / \partial t=$ 0 .

Based on these equations, it is thus possible to calculate the saltation matrix by using (5) and using that, the monodromy matrix of the system from (6). For $k=1$ the monodromy matrix is

$$
\mathbf{M}=\left[\begin{array}{cccc}
0.9996 & 0.0568 & -0.0055 & -0.0055 \\
-0.0075 & -0.4146 & 0.2931 & 0.2934 \\
-0.0050 & -0.0002 & 0.9950 & 0.0000 \\
-0.0000 & -0.0000 & 0.0014 & 1.0000
\end{array}\right]
$$

To further illustrate, we calculated the Floquet multipliers for the values of $V_{\text {in }}$ used in Fig. 3. The calculated eigenvalues are given in Table I, which shows that there is a period doubling bifurcation just before the value of $k=0.7$. This agrees with the results shown in Figs. 3 and 4. Note that in this specific system, the variables $x_{3}$ and $x_{4}$ do not influence the dynamics of $x_{1}$ and $x_{2}$ in each subsystem; they only play a role in deciding the switching instant. Another interesting property of this specific PFC topology is that 3 out of the 4 eigenvalues are very close to unity and they do not significantly change with changes in the bifurcation variable, and therefore they do not influence the analysis presented in the brief. 


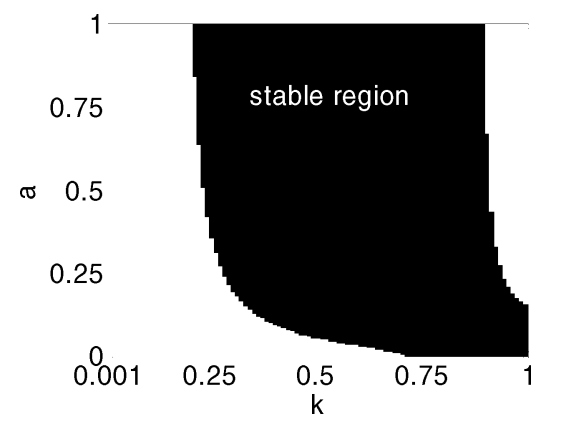

Fig. 5. Area in the $a$ versus $k$ parameter space for which the eigenvalues are inside the unit circle.

\section{Control of the Location of Period DOUBLING BIFURCATION}

From the structure of the monodromy matrix (6), it is clear that the saltation matrix $\mathbf{S}$ plays an important role in determining the stability of the limit cycle. Hence, it is possible to alter the stability property of the orbit by changing that matrix. From (5) it can be seen that the saltation matrix depends on the two vector fields (which we cannot change) and on the manifold $h$. Hence, by appropriately introducing small changes in $h$ it is possible to alter the stability properties of the system. We can either alter $h$ in such a way that the normal vector will change, or we can simply add a time varying component that will make the term $\partial h / \partial t$ nonzero. Thus, when the system tends to lose stability, we can force the eigenvalues back into the unit circle. This will extend the period one operation allowing us to control the parameter value where the first period doubling takes place.

To demonstrate this concept, we add a sinusoidal signal to $I_{\text {ref }}$ such that ${ }^{1}$

$$
I_{\text {ref }}=\left(x_{3}+x_{4}\right) P_{1} P_{2} V_{\text {in }}+a \sin \left(\omega t-\frac{\pi}{4}\right)
$$

where $\omega=2 \pi / T$. In Fig. 5 we plot the range of values of $a$ and $k$ where the eigenvalues are inside the unit circle. It is clear that for a wide range of supply voltages we can choose the value of $a$ such that the system is stable (notice that for very small values of $k$ we cannot stabilize the system). In both regions the system loses stability through period doubling bifurcation, which is verified by the corresponding eigenvalues as well as by cycle-to-cycle simulation. The graph leads to another interesting inference: that an incorrect value of $a$ can even destabilize a stable system. For example for $k=1$ and $a=0$ the system is stable but if $a$ is wrongly chosen at 0.5 then we have an unstable system. This underscores the need for a proper design of the controller rather than using trial and error techniques.

Now the question is, can we optimally choose the value of $a$ so that over a large range of $k$ the stability characteristics remain the same as that for $k=1$ ? We note that for $k=1$ the first eigenvalue is -0.41413 . In order to keep the eigenvalue fixed at that value, the optimal value of $a$ can be obtained by solving the nonlinear equation

$$
\left|-0.41413 \times \mathbf{I}_{4}-\mathbf{M}\right|=0 .
$$

${ }^{1}$ The injection of a sine wave has also been used in [17] where it was empirically found that it can stabilize $\mathrm{dc}-\mathrm{dc}$ buck converters.

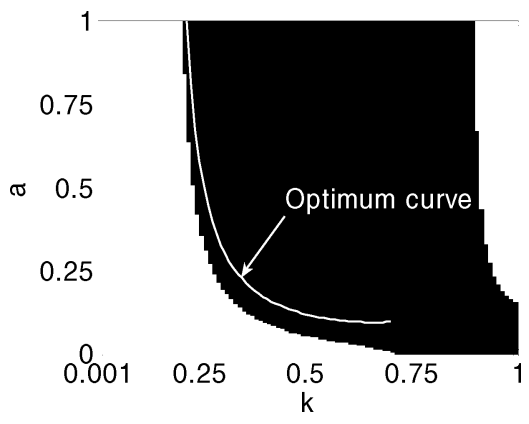

Fig. 6. Optimum values of $a$, only positive values are accepted.

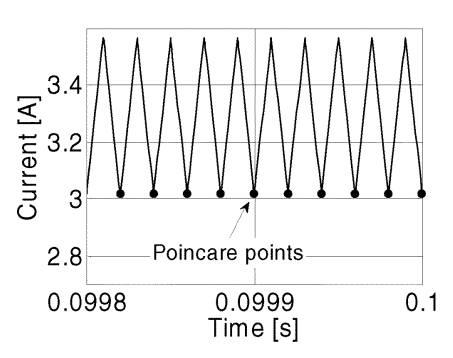

(a)

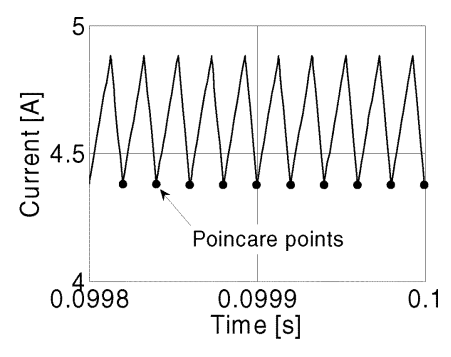

(b)
Fig. 7. Current response of the supervised system for (a) $\mathrm{k}=0.7$ and (b) $\mathrm{k}=$ 0.5 .

The result of numerical solution of this equation is plotted in Fig. 6.

On the basis of the above observation, we propose a supervising controller that adjusts the value of $a$ depending on the supply voltage by using a simple look-up table derived from (11). Thus, we can achieve a stable period- 1 behavior even when $V_{\text {in }}$ is time varying as in the case of the PFC. Fig. 7 shows the inductor current waveforms of the supervised system for $\mathrm{k}=0.7$ and 0.5. Comparing with Fig. 3(c) and (d) it is clear that the instability is avoided. Fig. 8 shows the sampled values of the inductor current of the system when $V_{\text {in }}$ is the original rectified sinusoid. It is clear that the overall performance of the system is greatly improved. To further test the robustness of the controller a sudden disturbance was imposed on the system in which the load was increased by $50 \%$ and as it can be seen from Fig. 9 the system remained stable (after the initial transient of course).

Fig. 8(a) shows that for very small values of $V_{\text {in }}$ the controller could not stabilize the system. It is possible to introduce other parameter changes [18] where the system performance could be further improved. For example, it is possible to alter the feedback variables and to introduce a two degrees of freedom controller which can stabilize the system while improving other performance characteristics like settling time and overshoot. Also it is possible to alter the amplitude and the phase of the injected sine wave which can guarantee even bigger stability regions.

\section{CONCLUSION}

In this brief, we have analyzed the stability of the PFC system with a peak current controlled boost converter. We have used a method of stability analysis based on the calculation of the state transition matrix over a complete clock cycle that also suggests a way of improving the stability margin. The monodromy matrix is composed of the state transition matrices during the on and off periods, and the state transition matrix across the switching event, called the saltation matrix. The calculation of 


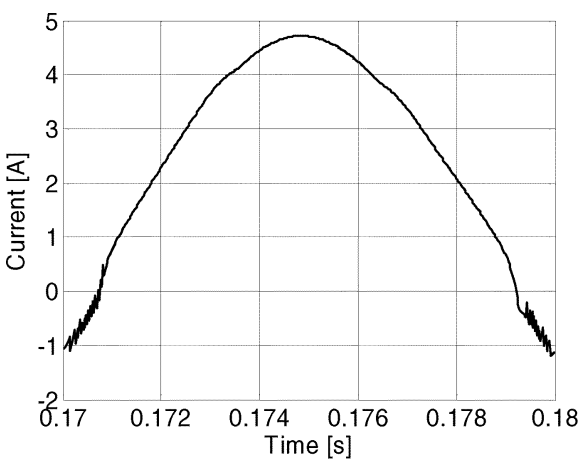

(a)

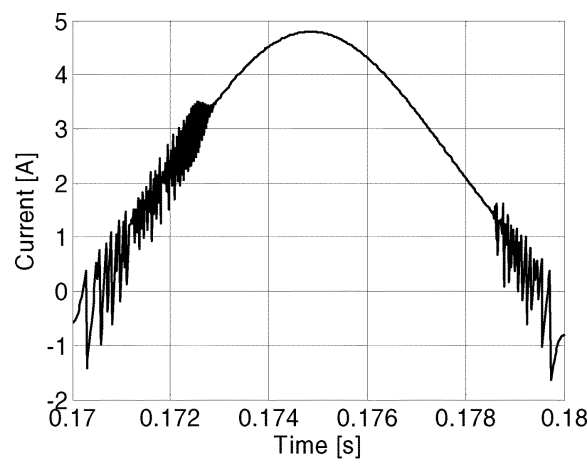

(b)

Fig. 8. Sampled inductor current of the system, a) with the proposed controller, b) without the proposed controller.

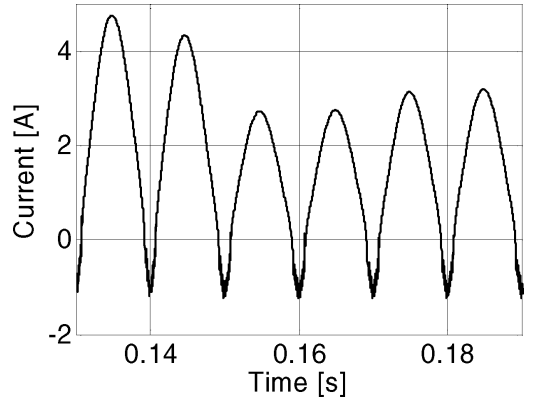

Fig. 9. Sampled inductor current response of the supervised system when subjected to a sudden disturbance.

the eigenvalues of the monodromy matrix (or the Floquet multipliers) shows that the system loses stability through a period doubling bifurcation when the input voltage to the boost converter goes below 0.7 times the peak voltage, thus degrading the system performance. Further reduction of the input voltage results in the occurrence of a succession of smooth and nonsmooth bifurcations which cause the system to behave chaotically. We propose a control strategy aimed at manipulating the saltation matrix - a major determinant of the stability — by adding a sinusoidal signal to the reference current. We show that it is possible to optimally choose the amplitude of the added sinusoid depending on the input voltage to place the eigenvalues at a convenient position. The resulting supervising controller, which is easily implementable on digital signal processor, greatly increases the parameter range for stable period-1 operation, and improves the overall performance of the PFC.

It may be noted that the technique of injecting a sinusoidal waveform to stabilize a converter circuit, referred to as resonant chaos control, has been tried by other researchers [17]. This earlier work was mainly empirical in nature, and the controller design was achieved through trial and error. In this brief we have shown the theoretical basis behind the success of this technique, proved that a wrong parameter choice can in fact destabilize the system, and proposed a semi-analytical method for the optimal choice of the controller parameters.

\section{REFERENCES}

[1] S. K. Mazumder, A. H. Nayfeh, and D. Boroyevich, "A novel approach to the stability analysis of boost power-factor-correction circuits," in Proc. IEEE Power Electron. Specialists Conf., Vancouver, BC, Canada, 2001, pp. 1719-1724.
[2] M. Orabi, T. Ninomiya, and C. Jin, "New formulation for stability analysis of power-factor correction converters," in Proc. VIII IEEE Int. Power Electron. Congr., Guadalajara, Mexico, 2002, pp. 33-38.

[3] X. Wu, C. K. Tse, O. Dranga, and J. Lu, "Fast-scale instability of singlestage power-factor-correction power supplies," IEEE Trans. Circuits Syst. I, Reg. Papers, vol. 53, no. 1, pp. 204-213, Jan. 2006.

[4] C. K. Tse, Complex Behavior of Switching Power Converters. Boca Raton, FL: CRC, 2003.

[5] Nonlinear Phenomena in Power Electronics: Attractors, Bifurcations, Chaos, and Nonlinear Control. S. Banerjee and G. C. Verghese, Eds. Piscataway, NJ: IEEE Press, 2001.

[6] S. K. Mazumder, A. H. Nayfeh, and D. Boroyevich, "Theoretical and experimental investigation of the fast- and slow-scale instabilities of a dc-dc converter," IEEE Trans. Power Electron., vol. 16, no. 2, pp. 201-216, Mar. 2001.

[7] M. Orabi and T. Ninomiya, "Nonlinear dynamics of power-factor-correction converter," IEEE Trans. Ind. Electron., vol. 50, no. 6, pp. 1116-1125, Dec. 2003.

[8] H. H. C. Iu, Y. Zhou, and C. K. Tse, "Fast-scale instability in a PFC boost converter under average current-mode control," Int. J. Circuit Theory Appl., vol. 31, pp. 611-624, 2003.

[9] O. Dranga, C. K. Tse, H. H. C. Iu, and I. Nagy, "Bifurcation behavior of a power-factor-correction boost converter," Int. J. Bifurc. Chaos, vol. 13, pp. 3107-3114, 2003.

[10] S. Bai, N. Huang, and A. Ioinovici, "Small-signal modeling and dynamic analysis of a novel ZVZCS three-level converter," IEEE Trans. Circuits Syst. II, Exp. Briefs, to be published.

[11] D. Giaouris, A. Elbkosh, V. Pickert, B. Zahawi, and S. Banerjee, "Control of period doubling bifurcations in dc-dc converters," in Proc. Int. Control Conf., Glasgow, U.K., 2006, CDROM.

[12] Y. A. Kuznetsov, Elements of Applied Bifurcation Theory. New York: Springer, 2004

[13] R. I. Leine and H. Nijmeijer, Dynamics and Bifurcations of Non-Smooth Mechanical Systems. New York: Springer, 2004.

[14] H. J. Dankowicz and P. T. Piiroinen, "Exploiting discontinuities for stabilization of recurrent motions," Dyn. Sys., vol. 17, pp. 317-342, Dec. 2002.

[15] H. J. Dankowicz and J. Jerrelind, "Control of near-grazing dynamics in impact oscillators," in Proc. Roy. Soc. London Ser. A, Nov. 2005, vol. 461, pp. 3365-3380.

[16] P. T. Piiroinen and H. J. Dankowicz, "Low cost control of repetitive gait in passive bipedal walkers," Int. J. Bifurc. Chaos, vol. 15, no. 6, pp. 1959-1973, 2005.

[17] Y. Zhou, C. K. Tse, S. S. Qiu, and F. C. M. Lau, "Applying resonant parametric perturbation to control chaos in the buck dc/dc converter with phase shift and frequency mismatch considerations," Int. J. Bifurc. Chaos, vol. 13, pp. 3459-3471, Nov. 2003.

[18] D. Giaouris, A. Elbkosh, S. Banerjee, B. Zahawi, and V. Pickert, "Control of switching circuits using complete-cycle solution matrices," in Proc. Int. Conf. Ind. Technol. ICIT, Mumbai, India, 2006, CDROM. 